\title{
Construction of Albumin-Coated 3D Allograft Based on Cone-beam CT Images
}

\author{
János KÓNYA ${ }^{1}$, Klaudia KULCSÁR ${ }^{2}$ \\ Dent-Art-Technik Kft., Győr, Hungary \\ ${ }^{1}$ labor@dentarttechnik.hu \\ ${ }^{2}$ kulcsar.klaudia@dentarttechnik.hu
}

\begin{abstract}
The 3D block products presented in this study are used in dental surgery to provide bone replacement for patients who do not possess a sufficient amount of bone tissue for implantation. If dental implants are supported by a proper amount of allografts, the mastication ability of the patient can be totally restored. The required bone replacement for the insertion of dental implants is a reconstruction utilising lyophilized human bone tissue treated with alveolar and mandibular odontological albumin. This study puts emphasis on the analysis, planning and processing of СВCT images, and on the machining and production threedimensional albumin-coated allograft. The study also extends to former and current bone grafting techniques, and provides a review on BoneAlbumin and the selection of suitable materials. This paper also investigates the domestic and international bone grafting market. Furthermore, it contains a case study and conclusions.
\end{abstract}

Keywords: 3D block, allograft, BoneAlbumin, 3D bonegraft, human bone.

\section{Introduction}

In oral surgery, bone block grafts presented in studies are utilised in bone replacement. They are used in areas affected by bone deficiency. They are designed for patients who do not possess a proper amount of bone tissue for the insertion of socalled conventional axisymetric dental implants. With a sufficient number of implants used for teeth replacement, it is possible to restore total chewing ability. With the help of bone block grafts, we are able to restore missing anatomical parts of the mandible and maxilla, resulting from bone loss, osteoporosis or accidents, in a well-planned way.

The manufacturing process starts with a CBCT image of a given patient delivered to our laboratory from an oral surgery clinic. It is followed by CBCT image analysis and the determination of bone deficiency to be replaced. Following this, the number, type and size of implants are decided. The next step of implementation is the modelling phase where virtual CBCT image processing, absent bone modelling, implant and permanent denture design takes place.

It is followed by a design process after which plans are verified by a medical specialist. The selection of bone material takes place after consultation and necessary changes of design.

This actually includes the preparation phase for manufacturing. One of the most important and determinative steps is the succeeding bone machining. It takes place in a so-called clean environment, after which the bone tissue is transported back to the tissue bank for further post-processing. The last step of the process is the operation, where the personalized bone block graft is implanted during oral surgery.

The uniqueness of detailed bone components presented in this study arises from virtual pre-operation design, special machining process, and albumin treatment. This albumin treatment reduces the trauma of operation, enhances and speeds up healing, and also shortens the duration of bone remodelling. Thanks to all these factors bone volume is recovered at the implanta- 
tion site. Thus, with the utilization of bone block grafts, the restoration of missing anatomical parts of the mandibula and maxilla can be carried out in a pre-planned way.

\section{Short summary of previous and cur- rent methods}

Synthetic bone grafts and grafts from animals or humans have become widespread for filling and replacing missing bone tissue. There are four available methods for changing the state of bone deficiency.

In the first method, bone tissue is prepared by bone machining for perfect fitting. Here plastic transformation of the patient's existing jawbone ridge takes place. The goal here is to facilitate insertion of the chosen implant.

The second method is bone grafting. Materials utilized for bone grafting can be divided into four different categories according to their origin.

Autograft - Bone obtained from the same individual receiving the graft;

Allograft - The donor and recipient are the same species, but they are genetically different;

Xenograft - Bone graft obtained from different species than the recipient;

Synthetic variants - Hydroxyapatite, bioceramic, beta-tricalcium phosphate, calcium sulphate.

According to the third method, bone machining and bone grafting are used and utilized simultaneously. In this case, oral surgeons practice lateral augmentation, sinus lifting and bone splitting during the surgical procedure. Possible bone grafting materials can be autograft, allograft, xenograft, or synthetic materials.

The fourth method is the so-called Guided Bone Regeneration (GBR). Within the confines of this method, resorbable and non-resorbable membranes are utilized [1].

The most commonly used grafting materials in oral surgery, as of today and former practice, are own bone tissue (bone chips or block grafts), synthetic calcium phosphate-based grafts, or granulated grafting materials of animal origin.

According to previous applications, the best quality bone grafting material is the patient's own bone tissue (autograft). However, its availability is quite limited as bone grafting is necessary for patients who have bone deficiency. Thus, the sufficient volume of bone tissue is not available. So in general, own bone tissue is implanted with other grafting materials as supplements.
In this case, own bone tissue is usually excavated from other or more areas. According to the necessity for small to large amounts of bone tissue, autograft is usually excavated from the site of operation or nearby areas.

Nevertheless, if an excessive amount of own bone tissue is needed, bone chips or block grafts excavated from the patient's hip bone are used for implantation.

In addition, albumin-treated allograft as a bone grafting material is starting to become wellknown and used in current practice. This is due to functional experiences and its operative success-rate.

This study focuses on its realization and opportunities in the following parts.

\section{Detailed presentation of the choice of right material}

\subsection{BoneAlbumin}

Orthopaedic surgeon and medical researcher, Dr. Zsombor Lacza PhD and the leader of the workgroup responsible for the development of the new procedure are investigating BoneAlbumin human bone allograft. Research activity has been going on since 2006. Initially, material testing and animal experiments were carried out. They were followed by the first implantations into humans after obtaining the necessary permissions. After successful laboratory, clinical and histological results, the OrtoSera Dental company was established. Dental, oral surgical and implantological experiments started in 2015. [1].

"Conventional allografts, xenografts and synthetic materials are all lower quality compared to own bone tissue in terms of their material properties. Therefore both procedures (own bone tissue and bone from other sources) have their advantages and disadvantages” [2].

„A new technique seeks to utilize the advantages of these two possible procedures. In doing so, an alloplastic material is produced from the orthopedically excavated bone tissue of a previously examined and living human being. It goes through an albumin treatment after proper processing. BoneAlbumin created this way has become part of the available toolbox of dental implantology as a material with significantly different properties compared to former solutions. (Skaliczki et al. 2013; Klára et al. - 2015; Horváthy et al. - 2016; Schandl et al. - 2016)” [1]. 
Thanks to different experiments and studies, it has been found that BoneAlbumin has numerous positive effects and advantages. It has been proven to be an active substance, has a significant effect on regeneration, speeds up osseointegration, provides favorable surface for progenitor cell adhesion, induces further albumin production, reduces and blocks proliferation of bacteria and pathogens.

\subsection{Material}

Specialists, led by Dr. Lajos Csönge, at the Western Hungarian Regional Tissue Bank located at Győr County Hospital, have investigated the properties and behavior of albumin treated human allograft. Examinations of bone physiology and histology were carried out. Certain types of bone grafts were investigated including the main steps of their preparation. According to their conclusion, an ideal bone donor should be no older than 40 years. For the preparation in turn, spongiosa or corticospongiosa parts are needed. Particle size of bone graft granules can vary: smaller than $0,5 \mathrm{~mm}$; between 1,5 and $2 \mathrm{~mm}$; larger than $2 \mathrm{~mm}$. Bone block grafts can be produced in the previously defined sizes. During bone graft processing - according to Urist protocol - bone tissue from human dissection is desantigenized, partly decalcified. Afterwards it goes through autolysis and chemical sterilization. It is followed by the addition of albumin to the dissection. Osseoconductive, osseoinductive and osteogenesis inductive effects of BoneAlbumin enhances bone graft integration” [1].

As a matter of medical practice liberalization, Bone Albumin has been successfully utilized since 2010 in orthopaedics. It is also becoming even more widespread in the field of oral surgery since successful experiments were carried out in March 2015.

\subsubsection{Choice of proper material for 3 dimensio- nal, albumin-treated allograft designed using CBCT imaging}

AReplacement of bone tissue missing from areas of later implantation comes from alveolar and mandibular oral reconstruction, albumin-treated, lyophilized human bone tissue. Traumatological and orthopaedic surgeries have been successfulyly using human bone tissue for treating different types of bone deficiency in reconstructive operations for many decades.

Bone block graft preparation and the physiological method of final sterilization take place un- der controlled circumstances in the tissue bank of Petz Aladár County Teaching Hospital. Lypohipized bone tissues are embossed with an anatomical documentation marked on the packaging. They indicate the preparation state and structure of bone tissue, as well as the anatomical site of the donated bone part.

Petz Aladár County Teaching Hospital in Győr has the necessary ISO and MEES certificates for the preparation of the product, which means the preparation of bone tissue and its sterile packaging. Human bone tissue serving as the base material is delivered to the laboratory for further processing as shown in the Figure 1.

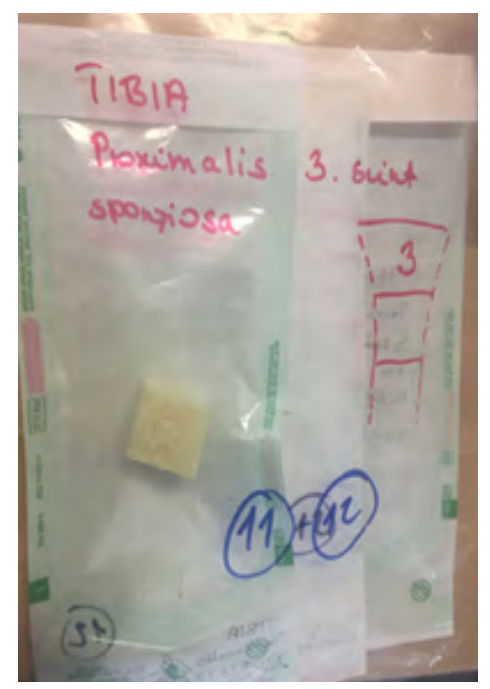

Figure 1. Embossed bone tissue part before ma-chining process

\section{Flowchart visualization and detailed presentation of the processing steps of bone block graft used in oral surge- ry - from the viewpoint of the dental prosthetics laboratory}

Designing and manufacturing process shown in Figure 2.

\subsection{Analysis}

The manufacturing process starts with the delivery of the patient's CBCT image from the contracting oral surgery clinic to our laboratory. It can be delivered online, by e-mail, by $\mathrm{CD}$, or by any other electronic data carrier. The layers and bone surfaces needed for designing the bone replacement are retrieved after a preliminary inspection carried out using CT analysis software. (Figure 3) 


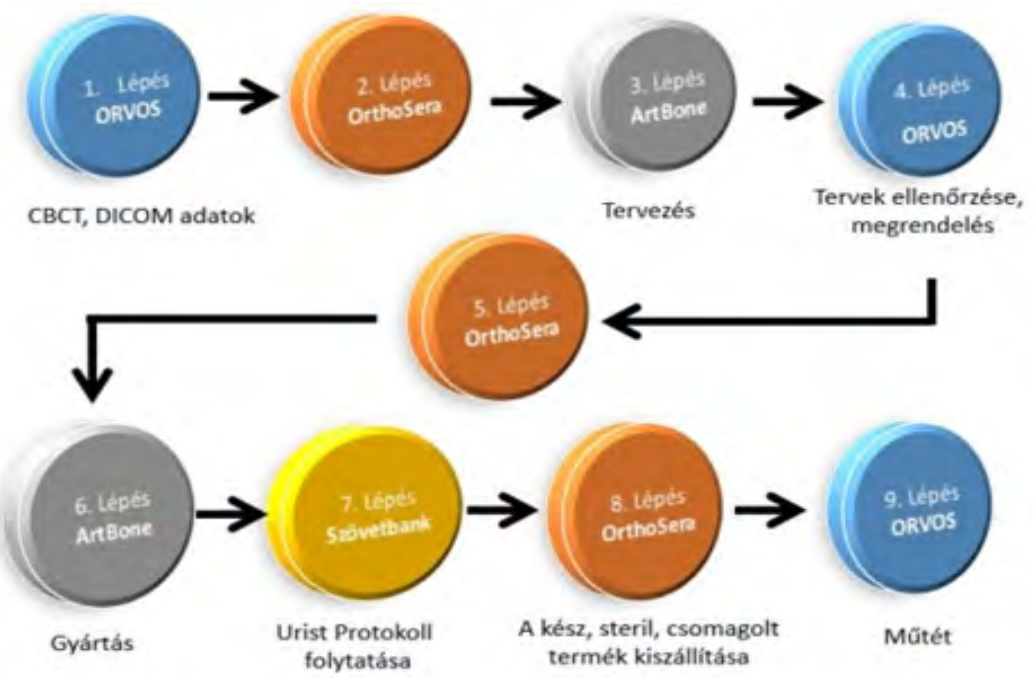

Figure 2. Flowchart of design and processing

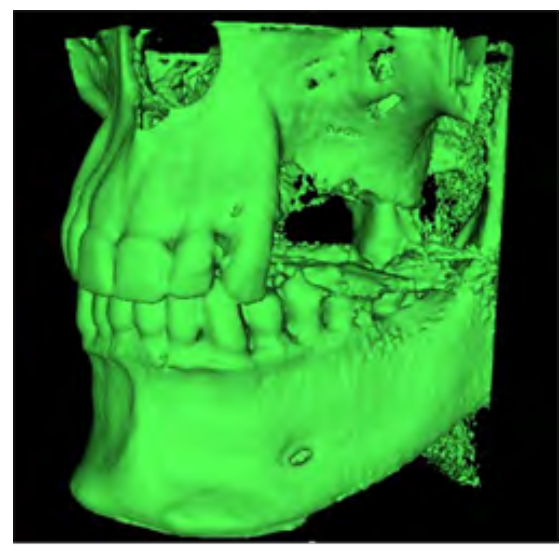

Figure 3. STL bone surface extracted from $C B C T$ image

This process is executed with two different software packages for the sake of the best solution. It is followed by the analysis in which the extracted bone surface is examined. Afterwards, bone deficiency to be replaced, number, type and size of implants to be used are discussed [3, 4].

\subsection{Virtual modelling}

During the modelling process, an ideal denture is designed, using a dental design software, onto the bone surface obtained from the CBCT image. Here, aesthetical, mechanical, functional and antagonistic aspects are considered (Figure 4). The number and type of implants were predominantly chosen by an oral surgeon. They are then

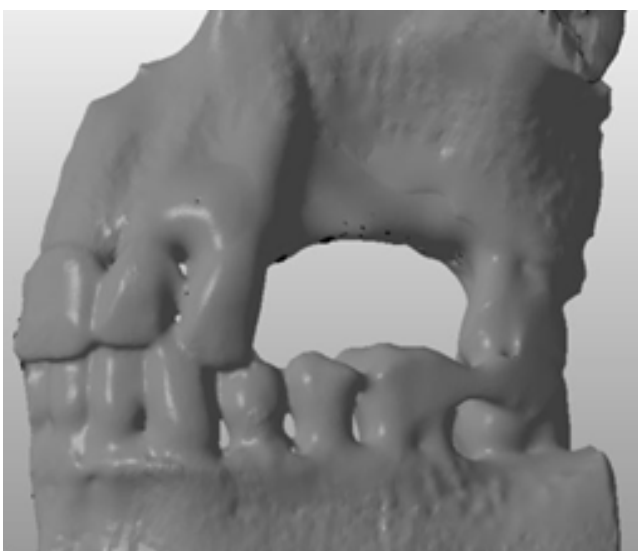

Figure 4. Virtually corrected STL bone surface

positioned with the utilization of the completed ideal denture (Figure 5) [5, 6].

\subsection{Design}

The complex 3D virtual model of the previously extracted bone surface and our design of ideal denture is created in another piece of design software (Figure 6). Several factors have to be considered during this design procedure. One of the most important and determinative aspects is the size of replaceable bone tissue and bone block graft. Perfect integration fully affects the design as probable bone loss has to be calculated when defining the geometrical sizes. Alongside these, another important aspect is the setting of the borderlines of the bone block graft. The so-called „nil 
nocere" principle is used for the determination of perfect borderlines. According to this, neighbouring teeth, existing bone dimensions, nerve and mucous membrane relations are considered [7-9].

With all the aspects mentioned above, the verification of design is performed in this phase by a medical specialist. It is also supplemented by a consultation with the contracting oral surgery clinic.

\subsection{Machining of bone block grafts}

The machining process starts with the manufacturing preparation of the verified bone part with a given geometry and volume (Figure 7). It takes place with the selection of the trabecular structure bone block graft of the proper size for machining. Implementation is carried out using a special microretentional surface fixing, which is developed by Dent-Art-Technik, without any processing aids. After virtual orientation setting, we place the workpiece into the zero point fixture of the 3D machining centre (Figure 8).

Machining takes place in a „clean” environment. The shaping of the bone block graft necessary for

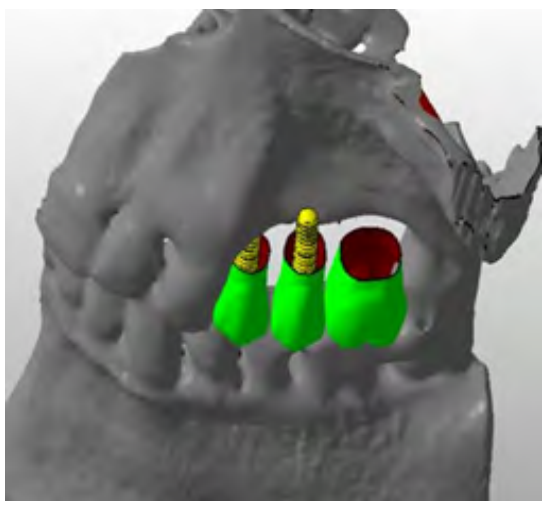

Figure 5. Design of the position of dentures and implants

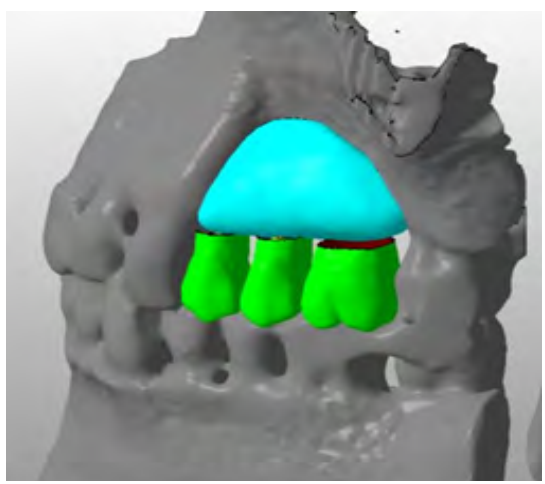

Figure 6. Designed bone replacement implantation is performed by clear water polishing with diamond-coated complex geometric tools. This procedure is the best and most suitable because, if machining was carried out by dry carving or milling, bone structure would be da-

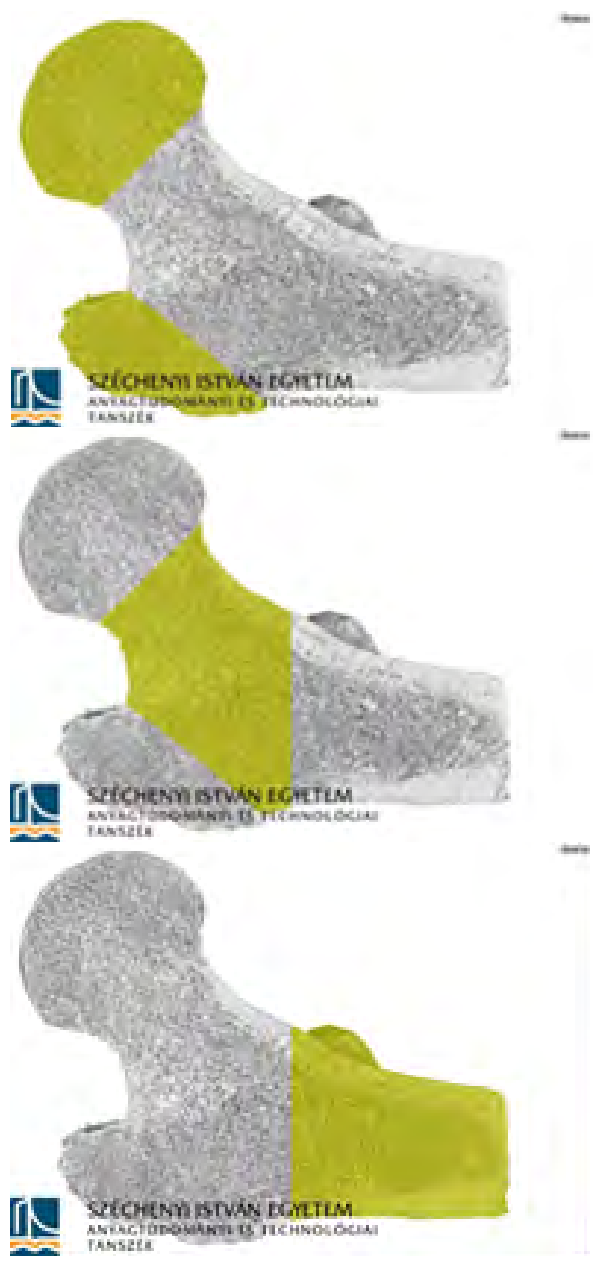

Figure 7. Classification of trabecular structure

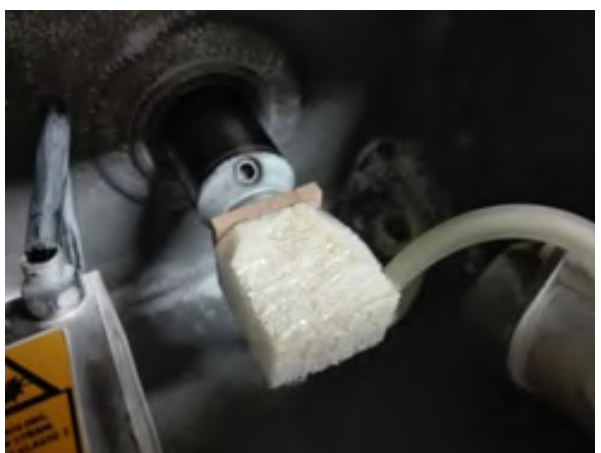

Figure 8. Bone block prepared for milling and placed into the machine 
maged considerably. The excessive erosion of the trabecular structure can be avoided by choosing polishing. The duration of this process is approximately 25-45 minutes.

The place of the lockpin is removed from the shaped bone part after machining. Then, nesting and insertion is checked under live circumstances with the help of a 3D-printed sterilizable model of the bone surface (Figure 9).

Finally, the bone block graft is packaged and equipped with the proper documentation. It is then transported to the tissue bank for post-processing steps such as cleaning, sterilizing, freeze-drying and diffusion albumin coating.

\subsection{Operation}

There is no need for significant bone surface corrections during the operation procedure when using the 3D-designed and machined bone block graft detailed presented in this study. This is because of the virtual design, which makes the high-precision bone block graft fit perfectly and immediately to its position. Fixation on the recipient site, which is the area of bone deficiency, is carried out with titanium microscrews. These are to be removed at the time of the insertion of subsequent implants (Figure 10, 11).

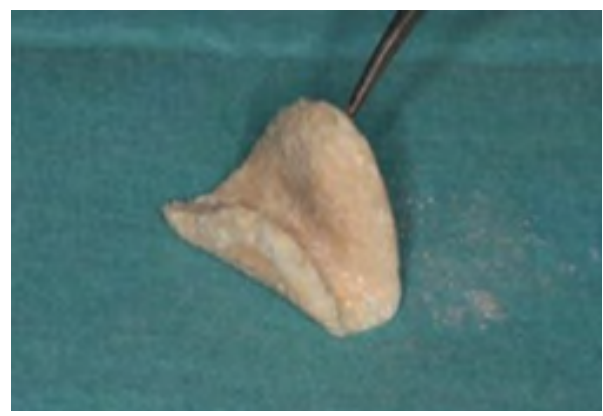

Figure 9. Machined bone block graft

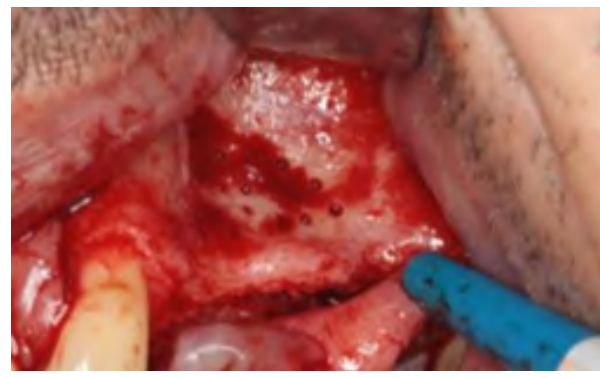

Figure 10. Recipient area perforated before im-plantation (Dr. Csaba Csák)
Vascularisation and integration of the bone block graft becomes considerably faster and safer as nesting and contact occurs on a surface of large extent. During oral surgical operations like this (when the allograft presented in this study is implanted), it is not required to add additional granules to the bone replacement material.

This is because the procedure is safer and faster compared to the alternative solution, when own bone tissue excavated from the patient serves as a grafting material. However, it is of vital importance during this procedure that the wound is closed with a tensionfree flap of mucosal membrane by the end of the operation. Another important medical factor is necessity for antibiotic protection (Figure 12, 13).

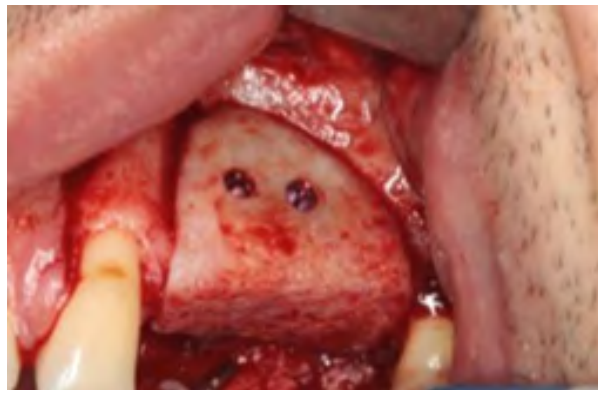

Figure 11. Inserted and fixed bone block graft (Dr. Csaba Csák)

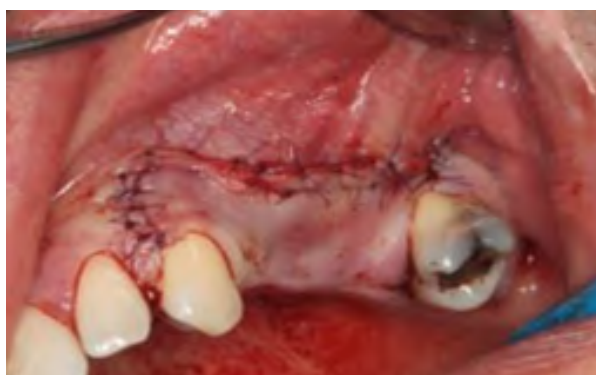

Figure 12. Surgical site closed with a tension-free flap (Dr. Csaba Csák)

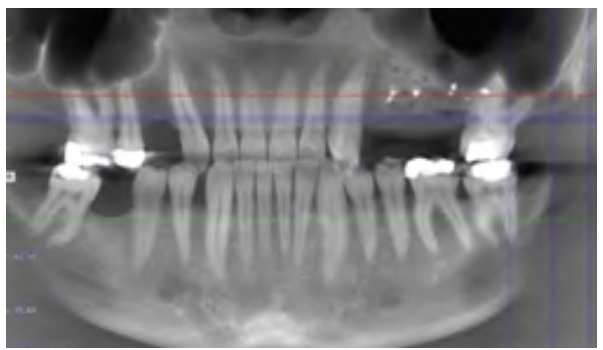

Figure 13. CBCT control after surgery (Dr. Csaba Csák) 


\section{Domestic and international market situation of bone parts presented in this study}

Our product occupies a special situation. Its appearance on the international market depends on the medical and donation laws of any given country. As our product is created from the processing of the bone tissue of a human donor, in addition to the previously mentioned aspects, religious interpretations can also limit its use.

Hungary was one of the first countries where bone tissue utilization of this kind was liberalized.

In the external market, a German company (Botiss) is producing similar products. They are also manipulating with bone tissue from living donors (they use remaining free bone tissue from femoral surgeries). This company does not accommodate any kind of additive that enhances tissue-growth, stimulates host responses, or generates integration. With the utilization of stimulating aids, the clinical integration of the bone block graft shaped and manufactured by us, is faster and more efficient.

The base material of the bone block graft is manufactured by many foreign companies, but the proper 3D machining is carried out by one company at a European level.

Our primary goal is the satisfaction of the domestic market. Hungary has a spotlight site in dental tourism, so the number of implantation procedures is high. Our worked-out surgical bone replacement aid can further increase the probability of implant insertion, stability and durability.

Product distribution is carried out by the company possessing the patent of albumin treatment. Its appearance on the international market, its widespread advertising, and the demonstration of the potential in the product has started throughout Europe. Legal regulations and sceptical opinions will be changed after the increasing number of successful implantation procedures.

The product is in the development and experiment phase. Its positive results inspire us for further development and technological optimization. We aim to improve and expand our product portfolio with surgical templates helping the latter insertion of implants, and with the production of immediate temporary restorations related to this. We are still in the initial phase of market uptake, but our research activities and surgical results in this topic have drawn a strong interest.

\section{Conclusions}

As presented in this study, this new bone grafting technique has several new possibilities. It has many advantages compared to other bone grafting procedures; it is essentially a modern and innovative technique. The advantages of the production of albumin-treated, three-dimensional allograft, designed using СВCT imaging, are due to the positive properties of BoneAlbumin and the wide spectrum of possibilities provided by virtual design.

One of the most important advantages of human allograft is its perfect tissue integration. Due to this, bone integration and regeneration begins earlier. This favourable process results in possible bone remodelling even in 3 months. Thus, the created bone replacement area becomes capable of accommodating the implant. After the integration of bone block graft, the created bone structure is almost identical to the structure of the patient's natural bone. It presents an ideal value of bone density, hardness and strength.

Another advantage to be mentioned is the low demarcation on graft-host interface. For the patient, it is of vital importance that the post-operative pain is much milder. However, it is also not negligible that the application is much easier and faster during the operation.

As it is not necessary to shape the bone block graft to the recipient surface during surgery, the time of surgical procedure decreases substantially. It is also not necessary to fix the membrane with pins, which further decreases operation time. Due to close connection and large contact surfaces between donor and recipient areas, vascularisation occurs faster and more securely.

Last but not least, another favourable property and important aspect is that there is no need for a second operation on the donor site. Thus, treatment time can be also reduced.

Another area of advantage lies in the wide spectrum of possibilities provided by virtual design. Virtual planning itself helps design and create the perfect implant model and the ideal denture. Using different types of dental, industrial, or general purpose software, the basis of virtual design is CBCT imaging.

One of the most important advantages of computer-aided design is the possibility to filter noise, shadows, image disturbances, and artefacts generated by the CBCT apparatus. It provides more precise surface matching for the bone replacement to be designed. We can optimally design the 
size and position of the implantable bone block graft for the patient due to the almost infinite possibilities provided by virtual design. The mobility of software is a further advantage as we are able to run different simulations on models. With all these, it becomes possible to evade probable unexpected consequences. It makes it possible to compare many good solutions and helps find the ideal one.

All in all, it can be stated that our revolutionary new biological technique of BoneAlbumin and the utilization of virtual design makes it possible for the unique-geometry bone block graft to restore the patient's chewing ability. In this way, the oriented, functional, prosthetic restoration of the patient comes to fruition. No less importantly, it improves the overall comfort of the patient.

\section{References}

[1] Gáspár L.: Csontmegmunkálás és csontpótlás az implantológiában. Dental Press Hungary Kft., Budapest, 2016.

[2] Gáspár L.: BoneAlbumin human allograft alkalmazásának tapasztalatai. Implantológia, 14/1. (2017) 46-56.

[3] Aranyarachkul P., Caruso J., Gantes B., Schulz E., Riggs M., Dus I., Yamada J. M., Crigger M.: Bone density assessments of dental implant sites: 2 . Quantitative conebeam computerized tomography. International Journal of Oral \& Maxillofacial Implants, 20/3. (2005) 416-424.

https://www.ncbi.nlm.nih.gov/pubmed/15973953

[4] Shahlaie M., Gantes B., Schulz E., Riggs M., Crigger M.: Bone density assessments of dental implant sites: 1. Quantitative computed tomography. In- ternational Journal of Oral \& Maxillofacial Implants, 18/2. (2003) 224-231.

https://www.ncbi.nlm.nih.gov/pubmed/12705300

[5] Gonda T., Kamei K., Maeda Y.: Determining favorable maxillary implant locations using three-dimensional simulation software and computed tomography data. International Journal Prosthodontics, 30/1. (2017) 58-61.

https://doi.org/10.11607/ijp.4972

[6] Moriwaki H., Yamaguchi S., Nakano T., Yamanishi Y., Imazato S., Yatani H.: Influence of implant length and diameter, bicortical anchorage, and sinus augmentation on bone stress distribution. Three-dimensional finite element analysis. International Journal of Oral \& Maxillofacial Implants, 31/4. (2016) 84-91. https://doi.org/10.11607/jomi.4217

[7] Kober C., Hellmich C., Stübinger S., Zeilhofer H. F., Sader R.: „Anatomical simulation” of the biomechanical behavior of the human mandible. International Journal of Computerized Dentistry, 18/4. (2015) 333-342.

https://www.ncbi.nlm.nih.gov/pubmed/26734667

[8] Oh T. S., Park J. S., Choi J. W., Kwon S. M., Koh K. S.: Risk factor analysis of bone resorption following secondary alveolar bone grafting using three-dimensional computed tomography. JPRAS 69/4. (2016) 487-492.

https://doi.org/10.1016/j.bjps.2015.11.002

[9] Kilinç, Y., Erkmen E., Kurt A: Biomechanical evaluation of different fixation methods for mandibular anterior segmental osteotomy using finite element analysis, Part two: Superior repositioning surgery with bone allograft. Journal of Craniofacial Surgery, 27/1. (2016) 36-40. https://doi.org/10.1097/SCS.0000000000002173 